\title{
A Comparative Study Between Whole Body Magnetic Resonance Imaging and Bone Scintgraphy In Detection of Bone Metastases In Patients With Known Breast or Lung Cancer \\ Wafaa Raafat Ali Abdel Hamid; \\ Department of Radiodiagnosis, Ain Shams University
}

\begin{abstract}
Purpose: The aim of this work is to compare the ability of whole body MRI including diffusion study with that of $99 \mathrm{~m}$ Tc-Methylene Diphosphonate Scintigraphy to detected skeletal metastases in patients with breast and lung cancer.

Patients and methods: 60 patients comprising 38 females and 22 males ranging in age from 30 to 60 years with a mean age of 48.1 years (mean age 47.3 years for females and 49 years for males) were enrolled in the study. 29 females are histopathologically proven cases of breast malignancy, 9 females and the 22 males are histopathologically proven cases of lung cancer.

The patients were referred from Oncological departments to perform the whole body MR study and bone scan at Ain Shams University hospitals MRI units and a private center during the time interval from December 2008 till December 2012.

All patients were subjected to both whole body MRI and bone scintigraphy. The whole body MRI was mainly obtained using 4 contiguous coronal stations for body coverage using the body coil and 2 contiguous sagittal stations for the spine using T1W FSE and STIR sequences. 48 out of 60 patients toke IV contrast and post contrast T1W imagaes with fat suppression were taken. The MRI examinations were performed using a superconducting 1.5 Tesla magnet (Achieva: Philips Medical Systems).
\end{abstract}

Standard skeletal Scintigraphy was performed using a planar one phase technique (delayed phase). The examination was done 2-3 hours after IV injection of technicium 99m labeled Methylene Diphosphonate with a maximum dose of $20 \mathrm{mCi}$.

Results: 42 patients out of 60 were positive for metastases based on histopathological verification or follow up. On MRI, 39 patients had metastases, 3 were false negative, 12 were true negative and 6 were false positive. On bone scan, 35 were true positive, 7 were false negative, 4 were false positive and 14 were true negative.

Based on lesion detection, on comparing bone scan to WB-MRI with and without diffusion, bone scan had an overall sensitivity of $85.6 \%$, specificity $67.5 \%$, PPV $61.7 \%$ and NPV $66.3 \%$, while WB-MRI had statistical values of $73.4 \%, 68.8 \%, 78.1 \%$ and $62.6 \%$ respectively.

On adding DWI, values raised to $88.9 \%, 82.9 \%, 93 \%$ and $69 \%$ respectively.

Quantitative analysis of DWI was also included in this study revealing an overlap between the Low ADC values of red marrow and metastasis at the range of 0.61 to $0.69 \times 10^{-3} \mathrm{~mm}^{2} / \mathrm{sec}$ and then between metastasis and other benign pathological marrow entities at the range of 0.9 to $1.03 \times 10^{-3} \mathrm{~mm}^{2} / \mathrm{sec}$.

WB-MRI also detected 64 soft tissue metastatic lesions which became 71 lesions on adding DWI with a distribution of hepatic, pulmonary, brain, adrenal, lymph nodes, pleural, leptomeningeal and renal lesions.

Conclusion: we don't think that WB-MRI should replace bone scan in the quest for skeletal metastases. However, we believe that WB-MRI should be the modality of choice when both skeletal and soft tissue metastases are suspected and in the follow up of a known metastatic patients for adequate coverage of both skeletal and soft tissue structures combined with qualitative and quantitative features of DWI helping assess tumors at cellular levels even prior to gross morphological changes verifying response to therapy as early as possible sparing patients precious time from drifting with delayed decision making. 


\section{Wafaa Abdel Hamid}

\section{INTRODUCTION}

It is well recognized that metastases to the skeleton is by far the most common type of malignant bone tumors. [1]

Evidence of osseous metastasis is of clinical importance because it is essential for clinical staging and the subsequent therapy planning. Localization and spreading of the skeletal metastasis must be displayed to identify possibly threatening complications in a timely manner. [2]

Metastasis of the bones tends to occur in areas of the red bone marrow with good vascularization, which, therefore, explains the tendency of metastases to be localized in the axial skeleton( $80 \%$ ), the femur $(40 \%)$ as well as the skull and the pelvis $(20 \%)$. Additional areas of manifestation are the ribs and the sternum (25-30\%). [3]

All existing methods of detecting skeletal metastases have certain limitations as discussed earlier in our study based on either the nature of the metastatic lesions (Lytic, sclerotic or mixed) or the distribution with certain blind spots encountered in each modality or severity of lesion (early or established with a spectrum of changes in between).

${ }^{99 \mathrm{~m}} \mathrm{Tc}$-phosphonate based skeletal scintigraphy has been the standard method for the initial staging of bone metastases. However, it depicts bone metastases at a relatively advanced stage of tumor infiltration when osteoblastic host reaction to tumor deposits has already occurred rendering it a fertile environment for a vast number of studies attempting to assess different parameters as conspicuity of lesions and the capability of different modalities to accurately characterize the lesions.

MRI has been verified to assess bone marrow metastases at significantly earlier stages than skeletal scintigraphy, yet due to the long examination time and the high cost, MRI has not been able to be widely applied as the routine method for the screening of the entire skeletal system, it has been confined to regional assessments based on equivocal findings on other imaging modalities or persistence of patient's symptoms despite negative findings on other modalities. Furthermore, there are technical limitations exists which have hampered fast MR imaging.[4]

New developments in gradient echo technology and new patient table and coil concepts have made MRI a method to be considered for the evaluation of tumor patients. [5]

Based on our results from the examination of 60 patients, whole-body MRI using T1W， STIR \& diffusion sequences through a moving tabletop and tabletop extender where patients were placed supine head first and were examined using the integrated body coil and an additional 32 channel phased array torso coil to improve signal arising from the chest and upper abdomen together with using a circularly polarized spine coil to receive high resolution spine images is a fast, dependable alternative to skeletal scintigraphy for the detection of metastases of the skeletal system.

Several studies have compared skeletal scintigraphy and WB-MRI reporting different sensitivities and specificities. Other studies tackled the additional role of DWI in further detection and characterization of skeletal lesions compared to established conventional MR sequences.

In our study we integrated both aspects WB-MRI compared to bone scan and the role of WB-DWI; the extra 25-30 minutes lengthening of the study, is it worth it?

\section{PATIENTS AND METHODS}

60 patients comprising 38 females and 22 males ranging in age from 30 to 60 years with a mean age of 48.1 years (mean age 47.3 years for females and 49 years for males) were enrolled in the study. 29 females are histopathologically proven cases of breast malignancy, 9 females and the 22 males are histopathologically proven cases of lung cancer.

The patients were referred from Oncological departments to perform the whole body MR study and bone scan at Ain Shams University hospitals MRI units and a private center during the time interval from December 2008 till December 2012. 
The inclusion criteria were: Age group: 30 to 60 years, both sexes are included, patients with histopathologically proven breast and lung cancer and current complaint of bone aches.

The exclusion criteria were: Children and elderly patients, lactating and pregnant females and patients known to have end stage renal disease.

\section{Bone scan imaging protocol:}

- Patients were informed to be well hydrated.

- Patients were asked to void immediately prior to study and frequently after the injection of the radiotracer administration throughout the day.

- Patients were asked to remove metal objects before imaging (e.g: watches, keys, jewelry, coins. etc)

- A $20 \mathrm{mCi}(70 \mathrm{MBq}$ ) bolus of Tc-99m MDP is injected intravenously.

- Imaging was commenced 2-4 hours after tracer administration.

- Anterior and Posterior images of the whole skeleton were taken at counts/image.

- Variable spot views were taken differing from one patient to another $(300 \mathrm{k}-500 \mathrm{k}$ counts/image).

\section{MR imaging protocol:}

Whole body MR studies were performed on 1.5-T superconducting magnet (Achieva; Phillips Medical Systems) by using a moving tabletop and tabletop extender.

The patients were placed supine head first and were examined using the integrated body coil and an additional 32 channel phased array torso coil to improve signal arising from the chest and upper abdomen. Images of the spine were acquired using a circularly polarized spine coil. Slice orientation was coronal for the trunk and extremities and sagittal for the spine. The entire MR imaging procedure took 60-90 min.

The following protocol was used for all patients, except for twelve patients who weren't administered IV contrast:
1) The parameters for the whole body coronal $\mathrm{T} 1$-weighted sequence were as follows:

single-shot turbo spin echo; TR/TE, 537/18; slice thickness, $6 \mathrm{~mm}$; gap, $1 \mathrm{~mm}$; number of slices, 30 per station; field of view, $530 \times 265 \mathrm{~mm}$; acquisition matrix, $208 \times 287$; number of signals acquired, 1 ; acquired voxel size, $1.27 \times 1.85 \times 6.00$ $\mathrm{mm}$; reconstructed voxel size, $1.04 \times 1.04$ $\times 6.00 \mathrm{~mm}$; acquisition time, 127 seconds/station, six stations total.

2) Applied parameters for the whole body coronal STIR sequence were as follows:

single-shot turbo spin echo; TR/TE, 444/64; inversion time, 165 milliseconds; slice thickness, $6 \mathrm{~mm}$; gap, $1 \mathrm{~mm}$; number of slices, 30 per station; field of view, 530 $\times 265 \mathrm{~mm}$; acquisition matrix, $336 \times 120$; number of signals acquired, 2; acquired voxel size, $1.58 \times 2.21 \times 6.00 \mathrm{~mm}$; reconstructed voxel size, $1.04 \times 1.04 \times$ $6.00 \mathrm{~mm} ;$ acquisition time, 58 seconds/station, six stations total.

3) Applied parameters for the whole spine sagittal T1W sequence were as follows:

turbo spin-echo images; TR/TE, 180/10; bandwidth, $195 \mathrm{~Hz} /$ pixel; number of slices, 11; section thickness, $4 \mathrm{~mm}$; intersection gap, $0.8 \mathrm{~mm}$; echo train length, three; refocusing flip angle, $180^{\circ}$; field of view, $380 \times 380 \mathrm{~cm}$; matrix, $177 \times$ 256; are acquired at two stations (cervicodorsal and dorsolumbar regions) and are composed into single images through computed alignment. The total imaging time for both stations is 2 minutes 12 seconds.

4) Applied parameters for the whole spine sagittal STIR sequence were as follows:

TR/TE, 4500/101; bandwidth, 252 $\mathrm{Hz} /$ pixel; acceleration factor, 2; number of sections, 11; section thickness, $4 \mathrm{~mm}$; echo train length, 29; refocusing flip angle, $150^{\circ}$; field of view, $380 \times 380 \mathrm{~cm}$; matrix, $320 \times 320$; are acquired at two stations (cervicodorsal and dorsolumbar regions) and are composed into single images by computed alignment. The total imaging time for both stations is 2 minutes 36 seconds. 
5) Applied sequence parameters for axial DWI were as follows:

single-shot spin-echo echo-planar imaging; TR/TE,612/78; inversion time, 180 milliseconds; slice thickness, $4 \mathrm{~mm}$; gap, $0 \mathrm{~mm}$; number of slices, 60; field of view, $450 \times 360 \mathrm{~mm}$; acquisition matrix, $128 \times 81$; receiver bandwidth, 1,874 $\mathrm{Hz} /$ pixel; motion-probing gradients in three orthogonal axes; $b$ values, 0 and $1,000 \mathrm{~s} / \mathrm{mm}^{2}$; number of signals acquired, 3; half-scan factor, 0.651; parallel acquisition (sensitivity encoding) factor, 2; echo-planar imaging factor, 43; acquired voxel size, $3.52 \times 4.50 \times 4.00 \mathrm{~mm}$; reconstructed voxel size, $1.76 \times 1.76 \times$ $1.76 \mathrm{~mm}$; acquisition time, 4 minutes 4 seconds/station, with a total of 5 stations. The total actual acquisition time for whole-body DWI was approximately 2025 minutes.

Coronal MIP's and 3D MPR's were generated.

6) During contrast-enhanced wholebody MR examination, a standard dose (0.1 mmoL per kilogram of body weight) of contrast material was administered intravenously through the antecubital vein and delayed fat suppressed T1W images were acquired.

Data Analysis:

On MRI, a skeletal lesion of high signal intensity on STIR image and low signal intensity on $T_{1}$ weighted images with or without avid post contrast enhancement was considered to be a suspected metastasis furtherly correlated with restricted diffusion and altered ADC values to establish the diagnosis. Areas of abnormal uptake of ${ }^{99 \mathrm{~m}}$ Tc-MDP on skeletal scintigraphy that could not be definitely explained by benign abnormalities, e.g. degenerative joint disease, secondary changes to fractures were considered to be suspected metastases.

A patient was considered to have negative imaging findings when no bony lesions meeting the criteria of malignancy were identified. Findings of the whole-body MRI and the skeletal scintigram were compared to the "gold standard" which was either biopsy and histopathological verification (not feasible for all lesions) or follow up (at a 3-6 month interval).
Another parameter was assessed which is Conspicuity and contrast-to-noise ratio to analyse and compare the different MR sequences (T1W, STIR and DWI):

The MR imaging sequences were compared qualitatively by ordering the sequences according to the conspicuity of the bone metastases and semiquantitatively in terms of contrast-to-noise ratio (CNR).

CNRs were computed according to the following formula: $\mathrm{CNR}=$ (SImet SIbone)/N, with SImet and SIbone defined as mean signal intensity inside the ROI placed on the metastasis and normal bone, respectively, and $\mathrm{N}$ defined as noise signifying the standard deviation inside the ROI placed outside the body.

Soft tissue lesions detected on MRI were compared in terms of detection and characterization before and after adding DWI.

Statistical analysis: Sensitivity, specificity, PPV and NPV were calculated for lesion detection and characterization by bone scan, WB-MRI with and without diffusion and compared. The CNRs were compared with a general linear model with repeated measures. The effect of the innersubject factor was tested by using a difference with $\mathrm{P}<.05$ as significant. Calculations were performed by using a statistical software package (JMP 7.0.2, 2008)/McNemar test.

\section{RESULTS}

Our study included 60 patients comprising 38 females and 22 males ranging in age from 30 to 60 years with a mean age of 48.1 years (mean age 47.3 years for females and 49 years for males). 29 females are histopathologically proven cases of breast malignancy, 9 females and the 22 males are histopathologically proven cases of lung cancer as demonstrated in table (1).

Table (1): Distribution of the sixty patients in the different age groups.

All the patients were subjected to a whole body MR study including coronal T1W, STIR and DWIBS sequences, axial DWIs were acquired for the purpose of quantification (generating ADC maps), sagittal T1W and STIR images of the 
whole spine together with a post contrast study within 72 hours to a maximum of one week from scintigraphic bone scan during the time interval from December 2008 till December 2012.

Twelve cases out of sixty refused contrast as they couldn't tolerate the length of the study with their bone aches and they refused sedation.

The cases came presented primarily with regional bone aches, (some had accentuated regional bone aches on top of generalized bone aches) and other symptoms variably encountered including dyspnea , jaundice, disturbed consciousness level and /or other neurological manifestations.

The 29 breast cases enrolled in the study were in either of the following stages of management:

1) Recent diagnosis prior to commencement of therapy: 14 cases.
2) After surgery prior to commencement of CTH or RTH: 11 cases.

3) On CTH and RTH after surgery: 2 cases.

4) On hormonal therapy after stoppage of post surgical CTH and RTH: 2 cases.

5) On neoadjuvant $\mathrm{CTH}$ in a case of advanced breast malignancy: 1 case.

The 31 lung cases enrolled in the study were in either of the following stages of management:

1) On CTH and RTH without surgery: 15 cases.

2) Recent diagnosis prior to commencement of therapy: 10 cases.

3) On CTH after surgery: 6 cases.

The results of bone scans and whole body MR studies were either verified by follow up or by biopsy where 25 lesions were biopsied in 22 patients (14 breast cases and 8 lung cases) and the rest were followed up.

\begin{tabular}{|l|l|l|l|}
\hline Age & Male & Female & No of cases \\
\hline $30-40 \mathrm{yrs}$ & 8 & 11 & 19 \\
\hline $41-50 \mathrm{yrs}$ & 6 & 14 & 20 \\
\hline $51-60 \mathrm{yrs}$ & 8 & 13 & 21 \\
\hline Total & 22 & 38 & 60 \\
\hline
\end{tabular}

\section{BONE SCAN RESULTS:}

A total of 158 lesions were reported on bone scans, 71 lesions were vertebral, 43 lesions were pelvic, 21 lesions were thoracic, 16 lesions were in the appendicular skeleton and 7 were calvarial as plotted in the graph showing the abundance of vertebral lesions.

The lesions reported with confidence as metastatic included 59 vertebral lesions, 22 pelvic lesions, 15 thoracic lesions, 8 appendicular lesions and 7 calvarial lesions showing the prevalence of confidently detected vertebral lesions.

As for the equivocal lesions, they were predominantly seen along the appendicular skeleton falling in the differential diagnosis of metastases, assymetric patchy marrow hyperplasia and osteonecrosis.

Based on our study, the highest degrees of confidence in the detection of metastatic osseous lesions were encountered with thoracic metastasis followed by calvarial and vertebral metastases, while the least confidence and accordingly the more equivocal lesions were met with as regards the pelvic and appendicular lesions.

Out of these 111 confidently diagnosed metastatic lesions, 19 turned out to be false positive with a distribution as displayed in the pie chart (11 lesions confirmed by biopsy , 5 by disappearance/ appearing stationary without treatment on follow up studies and 3 confirmed not malignant on other imaging modalities).

There were 22 mismatched lesions between bone scan and MRI that were not seen on bone scan, 15 lesions were confirmed malignant; 6 through biopsy and histopathological verification and 9 through progression on follow up.

The majority of those 15 false negative lesions on bone scan were appendicular followed by pelvic lesions as plotted on the pie chart with a distribution of 4 pelvic ( 2 confirmed on biopsy \& 2 on follow up), 7 appendicular ( 3 confirmed on biopsy \& 4 on follow up), 2 vertebral ( 1 confirmed 
on biopsy \& 1 on follow up) and 2 thoracic lesions confirmed on follow up.

Collectively, the least fallacies including false positive and false negative interpretations were noted as regards the calvarial lesions, followed by vertebral lesions, pelvic lesions, thoracic lesions and ending with appendicular lesions.

Accordingly, sensitivity, specificity, PPV and NPV were calculated regionally to verify the accuracy of bone scan in detecting metastatic lesions based on their distribution as enclosed in table (2).

\begin{tabular}{|l|l|l|l|l|l|}
\hline & $\begin{array}{l}\text { Vertebral } \\
\text { lesions }\end{array}$ & $\begin{array}{l}\text { Pelvic } \\
\text { lesions }\end{array}$ & $\begin{array}{l}\text { Thoracic } \\
\text { lesions }\end{array}$ & $\begin{array}{l}\text { Appendicular } \\
\text { lesions }\end{array}$ & $\begin{array}{l}\text { Calvarial } \\
\text { lesions }\end{array}$ \\
\hline Sensitivity & $91.6 \%$ & $85.1 \%$ & $81.8 \%$ & $69.8 \%$ & $100 \%$ \\
\hline Specificity & $66.6 \%$ & $56.2 \%$ & $60 \%$ & $28.5 \%$ & $76.6 \%$ \\
\hline PPV & $91.9 \%$ & $76.6 \%$ & $81.8 \%$ & $58.3 \%$ & $80 \%$ \\
\hline NPV & $80 \%$ & $69.2 \%$ & $60 \%$ & $22.8 \%$ & $100 \%$ \\
\hline
\end{tabular}

Table (2): Collectively demonstrating the sensitivities, specificities, PPV's and NPV's of bone scan in detection of osseous metastases based on their distribution.

\section{WHOLE BODY MRI RESULTS:}

A total of 172 osseous lesions were reported on MRI without diffusion with a distribution of 82 vertebral lesions, 43 pelvic, 33 appendicular, 10 thoracic and 4 calvarial lesions with an abundance of vertebral lesions.

Out of those 172 lesions, 112 lesions were reported malignant with confidence distributed as 62 vertebral lesions, 26 pelvic lesions, 18 appendicular lesions, 4 thoracic lesions and 2 calvarial lesions.

On adding diffusion, 197 lesions were detected with a distribution of 89 vertebral lesions, 50 pelvic, 38 appendicular, 16 thoracic and 4 calvarial lesions.

Out of which 134 lesions were reported malignant with confidence with a distribution of 70 vertebral lesions, 36 pelvic lesions, 21 appendicular lesions, 5 thoracic lesions and 2 calvarial lesions.

At first, the influence of adding DWI to WB-MRI is seen in the increase in number of lesions detected and is then emphasized through comparing the percentage of confidently detected lesions based on the opinion of an experienced MR reader with and without DWI.

After adding DWI, lesions that were confidently called metastatic raised to 134 lesions. As for the remaining 63 lesions; 54 were considered benign and 9 lesions were equivocal.

On comparing the equivocal lesions before and after adding DWI, 9 lesions remained equivocal, while the other 7 lesions were differentiated into 2 benign vertebral lesions with no diffusion restriction suggestive of osteoporotic changes furtherly confirmed by DEXA. The other 5 lesions were called metastatic attributed to their frank diffusion restriction with low mean ADC values, 3 were confirmed metastatic by biopsy from the right iliac bone, left femoral shaft and sacrum, as for the remaining 2 lesions, they were vertebral and remained unchanged on follow up for which they were considered false positive

Then there were the 25 lesions that were only seen on DWI's and not visualized on conventional MR images, 16 of which were not seen on bone scan, 17 of these lesions detected only on diffusion were true positive, 5 lesions were true negative and the remaining 3 were false positive.

There was another mismatch between MRI and bone scan with the lesions being inconspicuous on MRI.

20 lesions were inconspicuous on MR images (without diffusion) although demonstrated on Bone Scan and reported as malignant, 14 of which were verified to be false negative on MRI/true positive on Bone Scan; 1 was biopsied from a vertebra and histopathologically verified, the other 13 lesions were verified through follow up studies, either showing regression on post therapy follow up or progression on follow up without therapy), the other 6 lesions were verified as false positive; 2 by biopsy from femoral and pelvic lesions and the 
other 4 confirmed benign by follow up studies.

On adding diffusion, these mismatched lesions came down to 14 lesions that remained inconspicuous while the other 6 lesions included 3 vertebral metastatic deposits, faint diffusion restriction at a left femoral head that was confirmed on a follow up MR study to be early avascular necrosis and two other pelvic lesions that turned out to be patchy marrow hyperplasia. The fate of these lesions is demonstrated in table (19).

On WB-MRI without diffusion 91 lesions are true positive, 18 lesions are false

\begin{tabular}{|l|l|l|}
\hline & Bone Scan & \\
\hline Sensitivity & $85.6 \%$ & \\
\hline Specificity & $67.5 \%$ & \\
\hline PPV & $61.7 \%$ & 78 \\
\hline NPV & $66.3 \%$ & 62 \\
\hline
\end{tabular}

Table (2): comparing global sensitivies, specificities, PPV's and NPV's of bone scan, WB-MRI without diffusion and WBMRI with diffusion in detection of osseous metastases.

Quantitative analysis of the diffusion study showed variable degrees of overlap in ADC values between the different pathological entities.

There is an overlap between the Low ADC values of red marrow and metastasis at the range of 0.61 to $0.69 \times 10^{-3} \mathrm{~mm}^{2} / \mathrm{sec}$ and then between metastasis and other benign pathological marrow entities at the range of 0.9 to $1.03 \times 10^{-3} \mathrm{~mm}^{2} / \mathrm{sec}$ sparing the midrange of 0.7 to $0.89 \times 10^{-3} \mathrm{~mm}^{2} / \mathrm{sec}$ where only metastatic lesions gave these values in our study.

Biopsy helped in verifying the following 25 lesions (10 pelvic, 6 femoral, 6 vertebral, 2 tibial and 1 sternal) distributed as follows:

The forementioned results are representing one sector of the study which is considered the main sector, but then there is another parallel sector of major influence on patient's management and therapeutic planning which is related to the detection and verification of soft tissue metastatic involvement and comparing results of MRI with and without DWI.

Conventional MR images revealed:

1) 23 hepatic focal lesions in 14 cases. positive, 34 lesions are true negative, 14 lesions are false negative and 16 lesions are equivocal.

Then on WB-MRI with diffusion, 119 lesions are true positive, 13 lesions are false positive, 41 lesions are true negative, 11 lesions are false negative and 9 lesions are equivocal.

Statistical analysis in the form of sensitivity, specificity, PPV and NPV are compared between bone scan and WBMRI with and without diffusion highlighted in table (2).

\begin{tabular}{|l|l|}
\hline $\begin{array}{l}\text { WB-MRI without } \\
\text { diffusion }\end{array}$ & $\begin{array}{l}\text { WB-MRI with } \\
\text { diffusion }\end{array}$ \\
\hline $73.4 \%$ & $88.9 \%$ \\
\hline $68.8 \%$ & $82.9 \%$ \\
\hline $78.1 \%$ & $93 \%$ \\
\hline $62.6 \%$ & $69 \%$ \\
\hline
\end{tabular}

2) 19 lymph nodes suggested to be metastatic, 11 were axillary in 7 breast cases and 8 were hilar/mediastinal in 4 lung cases.

3) 9 suggested adrenal metastases in 7 cases of lung cancer with a confidence in diagnosing 7 of these lesions.

4) 2 cases were suggested to have leptomeningeal infiltrates, one spinal and one intracranial with the latter being merely conspicuous.

5) 1 case of breast cancer showed renal metastasis.

6) 2 cases showed lesions suggested to be intramuscular soft tissue metastatic deposits, two at left calf muscles in one case and one at medial muscular compartment of the left thigh in another patient.

7) 5 cases of breast cancer and 4 cases of lung cancer showed pulmonary nodules (about 16) suggested to be metastatic.

8 ) 5 cases ( 3 breast cases and 2 lung cases) showed uneven pleural thickening, 3 of which were suspicious of a metastatic nature and 2 were suggested to be benign post irradiation pleural thickenings.

9) 12 cases showed 18 brain lesions best demonstrated on post contrast images with about 12 being confidently diagnosed as metastatic.

On adding diffusion to conventional MR images, it had the following influence on 


\section{Wafaa Abdel Hamid}

the detection and characterization of soft tissue lesions:

1) - 31 hepatic focal lesions in 16 cases, 11 were breast cases and 5 were lung cases.

2) - 22 lymph nodes suggested to be metastatic, 12 were axillary and 1 supraclavicular in 7 breast cases and 9 were hilar/mediastinal in 6 lung cases.

3) 10 suggested adrenal metastases in 7 cases of lung cancer with a confidence in diagnosing 9 of these lesions.

4) 3 cases were confidently called leptomeningeal infiltrates, one spinal and two intracranial (one of which was merely conspicuous on conventional MR images).

5) 1 case of breast cancer with renal metastasis.

6) The 3 intramuscular soft tissue lesions that were seen on conventional MRI; the two left calf lesions still remained equivocal due to the overlap in ADC values between a chronic hematoma and a soft tissue.

7) 6 cases of breast cancer and 4 cases of lung cancer showed 23 pulmonary nodules.

8) 7 cases ( 5 breast cases and 2 lung cases) showed uneven pleural thickening, 3 were suggested metastatic appearing as an irregular hypointense rim on corresponding ADC maps giving a low mean $\mathrm{ADC}$ value.

9) The 12 lesions reported metastatic out of the 18 brain lesions on conventional MRI were supported metastatic on DWI.

The majority of soft tissue lesions were verified on follow up and only the left calf intramuscular lesions, the left renal metastasis and an adrenal metastasis were verified by biopsies.
WB-MRI with diffusion came superior to WB-MRI without diffusion in the detection of:

1)8 extra hepatic focal lesions (6 metastatic) and adding 2 more patients.

2)3 extra metastatic lymph nodes.

3)1 extra adrenal metastasis in a case with no other soft tissue metastases.

4)1 extra leptomeningeal metastasis and an extra case was added.

5)7 extra pulmonary nodules.

6) 2 extra cases with pleural thickenings.

Although DWI showed a high sensitivity (94\% \& 86\%) in the detection of lymph nodes and pulmonary nodules respectively yet it showed a low specificity $(55 \%$ \& $52 \%$ ) in the differentiation between benign and malignant lymph nodes and pulmonary nodules respectively.

Two of the confidently called metastatic pleural thickenings on MRI with and without diffusion turned out to be false positive and suggested to be post irradiation fibrosis based on a one year follow up stationary course.

This is an example of a case show osseous and soft tissue metastatic lesions; a 42 year- old female patient with recently diagnosed breast cancer was presented with lower back pain, right sciatica.

Her bone scan revealed osseous metastases at D1 and L2. On correlation with her WB-MRI, it showed osseous metastases at C5, D1 and L2. The L2 vertebral lesion showed an extra-osseous soft tissue component intra and retrospinal compromising L2/L3 neural foramen, implicating right L3 nerve root. Her MR study more so her DWI showed 2 metastatic pulmonary nodules as demonstrated in figures (1) and (2). 

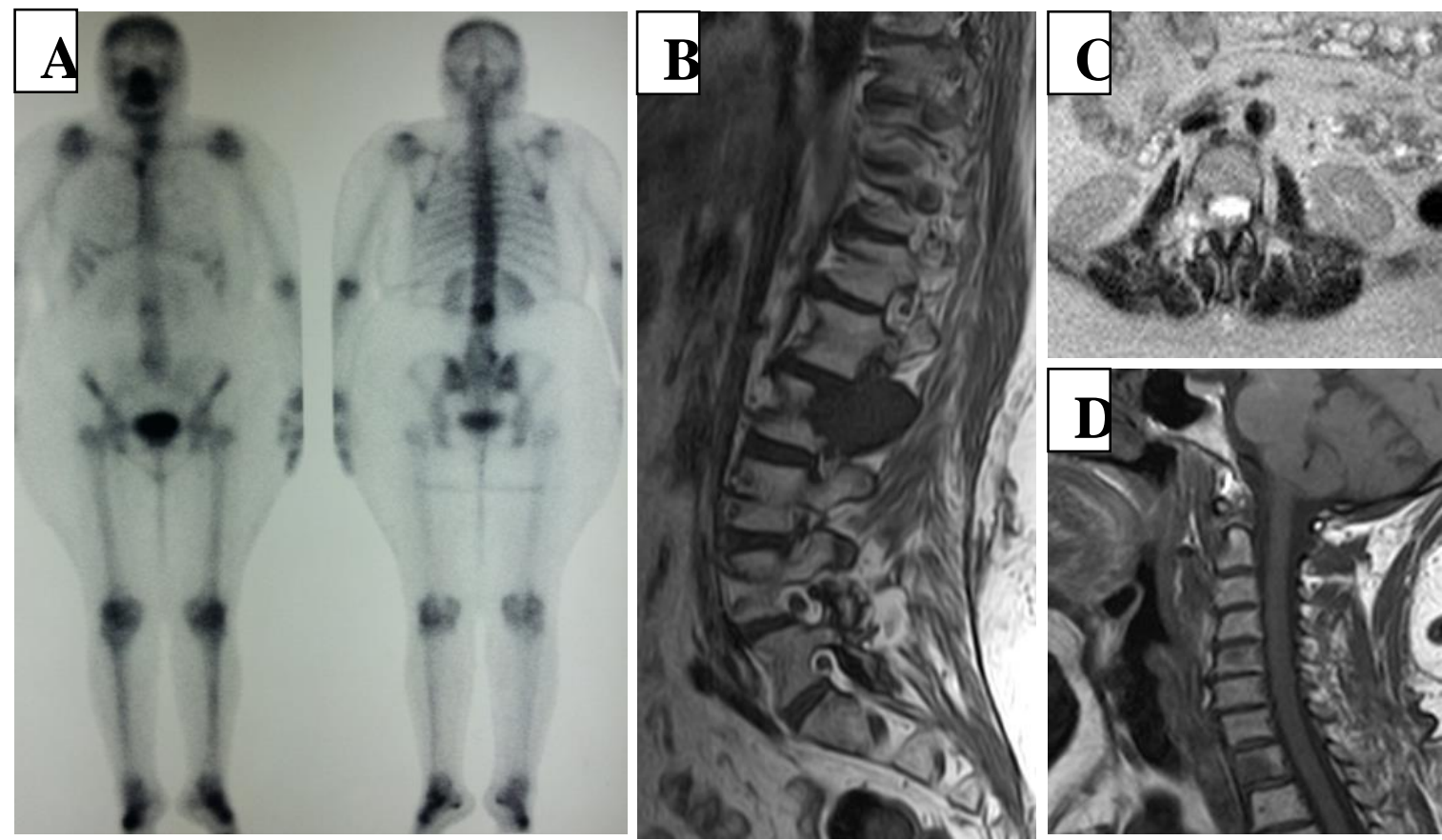

Figure (1): A case of metastatic breast cancer. (A) The patient's anter and posterior projections, bone scan showed focal zones of hot uptake at D1, L2 and right aspect of the manubrium sterni. (B) Sagittal T1W image of the dorsolumbar spine showed a sizable hypointense marrow lesion implicating the right posterolateral aspect of L2 vertebra and pedicle with expansion and extra-osseous soft tissue component furtherly demonstrated on (C) axial T2W image at L2 level showing the right sided infiltrative process with intra and retrospinal soft tissue extensions.(D) Sagittal T1W image of the cervical spine showing hypointense metastatic lesions at C5 and D1.

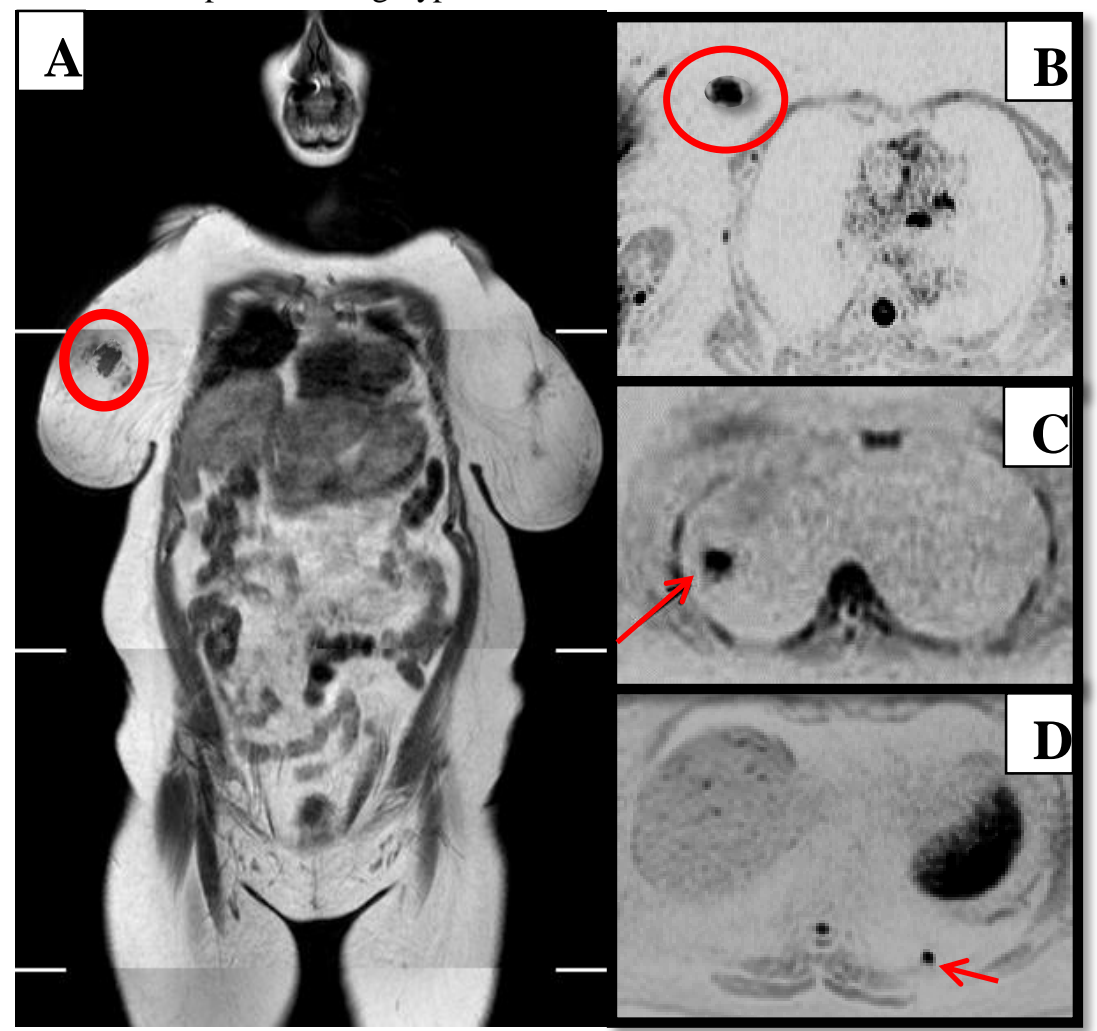

Figure (2): The same case of metastatic breast cancer showing (A) WB-MRI coronal T1W image showing the primary breast lesion seen at upper inner quadrant of the right breast exhibiting dark signal and showing irregular outlines. (B) Corresponding axial inverted DWI showing the breast lesion as a 
flared out zone of evident diffusion restriction, giving a mean ADC value of $0.91 \times 10^{-3} \mathrm{~mm}^{2} / \mathrm{sec}$. (C) \& (D) are also axial inverted DWI's at different levels showing the pulmonary nodules as rounded focal zones of diffusion restriction at lateral segment of right middle lung lobe $(\mathrm{C})$ and posterior segment of left lower lung lobe (D) giving a low mean ADC value ranging between 0.97 and $1.03 \times 10^{-3} \mathrm{~mm}^{2} / \mathrm{sec}$. In this case we can see that bone scan detected a sternal metastasis not conspicuous on MRI confirmed on follow up and the WB-MRI with diffusion showed a small lesion at C5 vertebra, a soft tissue component in association with the L2 lesion implicating right L3 nerve root and two pulmonary metastatic nodules.

\section{DISCUSSION}

Based on our study, the confidence of detection and characterization of skeletal metastases on bone scan was scaled as thoracic including rib and sternal lesions being the highest $(93.7 \%)$ with the least percent of equivocal lesions (5.2\%), followed by vertebral and calvarial lesions (86\% \& $87.5 \%$ respectively) with a percent of equivocal lesions $(12.6 \% \&$ $12.5 \%$ respectively), then we have pelvic lesions $(72.7 \%)$ with a percent of equivocal lesions (26\%) and last we have the appendicular lesions (57\%) with a percent of equivocal lesions (35.4\%).

According to this, we are proposing that skeletal scintigraphy is much better established for the detection and characterization of thoracic lesions followed by vertebral and calvarial lesions all the way down to appendicular lesions where bone scan wouldn't be the study of choice when it comes to decision making in patient management.

This is furtherly translated through sensitivity, specificity, PPV and NPV calculated according to regional distribution of lesions, with the highest values noted for calvarial lesions $(100 \%$, $76.6 \%, 80 \% \& 100 \%$ respectively) followed by vertebral lesions (91.6\%, $66.6 \%, 91.9 \%$ \& $80 \%$ respectively), then pelvic lesions $(85.1 \%, 56.25,76.6 \%$ \& $69.2 \%$ respectively) and thoracic lesions $(81.8 \%, \quad 60 \%, \quad 81.8 \%$ and $60 \%$ respectively) being more or less comparable and last come appendicular lesions $(69.8 \%, 28.5 \%, 58.3 \& 22.8 \%$ respectively).

Another study addressing the accuracy of bone scan, concluded that it had a sensitivity of $88.4 \%$ in vertebral lesions, $74 \%$ in pelvic lesions, $66.9 \%$ in calvarial lesions, $61 \%$ in thoracic lesions and $48 \%$ in appendicular lesions. [5]

Ryoto et al's results were comparable to our study concluding that bone scan had a high diagnostic accuracy in vertebral and calvarial lesions followed by thoracic and pelvic lesions and last in long bones.[6]

One of these cases that had false positive lesions was rather interesting having a rare autosomal dominant bone disorder known as Osteopoikilosis where the bone scan showed hot foci at the left femoral head, the corresponding MR study showed multiple rounded sclerotic bone lesions of more or less the same size at both hip joint regions (acetabular bones and femoral heads) appearing hypointense on $\mathrm{T} 1 \mathrm{~W}$ images, hardly conspicuous on STIR showing no diffusion restriction as seen in figure (3).

What was interesting in this case was that the patient was complaining of persistent back pain and was a recently diagnosed case of lung cancer. His bone scan came negative for any vertebral metastases while his MR study showed a lumbar lesion that was called metastatic attributed to patient's symptomatology and being an uncommon site of involvement for osteopoikilosis and furtherly confirmed by biopsy. 


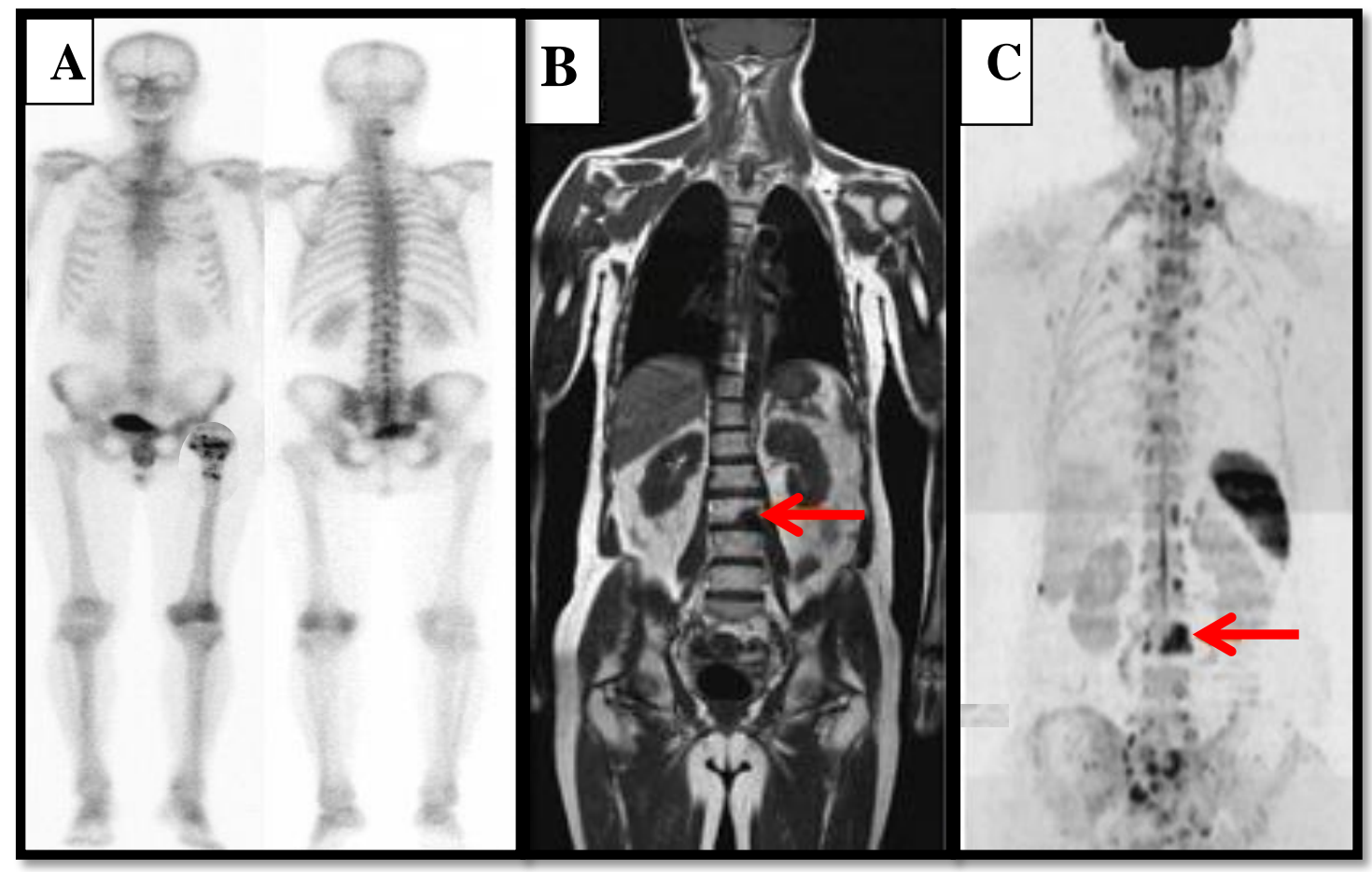

Figure (3): A known case of lung cancer for which patient was subjected to CTH and surgical resection and became tumor free for a year then started complaining of bone aches at his lower back and pelvis. (A) Anterior and posterior projections bone scan showed left femoral head enlargement with small focal zones of hot uptake reported metastatic. A small zone of warm up take is seen high cervical to the left and was suggested facetal arthropathy. (B) The patientls WB-MRI T1W coronal image showed an L2 metastatic marrow lesion exhibiting dark signal. It was not seen on bone scan furtherly demonstrated on 3D coronal MIP of the patients inverted DWI (C) showing a discreet focus of diffusion restriction to the left of L2 vertebra giving a low mean ADC value of $0.86 \times 10^{-3} \mathrm{~mm}^{2} / \mathrm{sec}$.

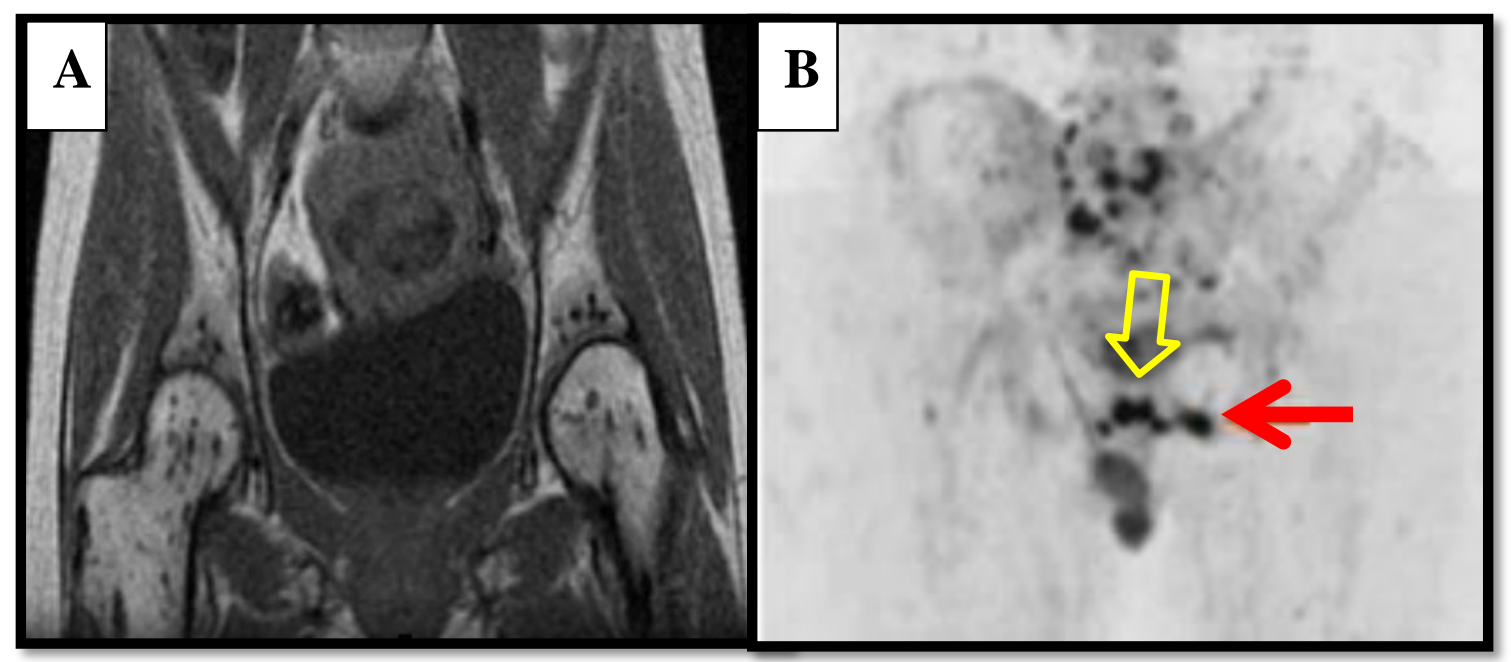

Figure (4): The same case showing (A) Coronal T1W image of the pelvis revealing multiple more or less same sized discreet foci of sclerosis at both acetabular bones and femoral heads typical for osteopoikylosis. Not seen on Coronal DWI of the pelvis (B) while a lesion showing diffusion restriction is demonstrated at left pubic bone (red arrow giving a low mean ADC value of $0.74 \times 10^{-3}$ $\mathrm{mm}^{2} / \mathrm{sec}$. There other zones of diffusion restriction corresponding to the symphsis pubis (yellow arrow) giving higher mean ADC values ranging between 1.03 and $1.12 \times 10^{-3} \mathrm{~mm}^{2} / \mathrm{sec}$ suggested to be secondary to ostieitis pubis. Those lesions were not conspicuous neither on conventional MRI nor on bone scan. 


\section{Wafaa Abdel Hamid}

The 15 false negative lesions on bone scan were predominantly lytic (11), two of which were aggressive pelvic and vertebral lesions. These lesions were encountered as 7 appendicular, 4 pelvic, 2 vertebral and 2 thoracic.

Collectively, we found out that bone scan gave an overall sensitivity of $85.6 \%$, specificity of $67.5 \%$, PPV of $61.7 \%$ and NPV of $66.3 \%$.

Those findings were partly noted in our study where most of the thoracic lesions (12 out of 15) were seen in breast cancer cases and then 5 of the 8 appendicular lesions delineated on bone scan were in lung cancer cases.

As for the WB-MRI study, it was assessed with and without diffusion to verify whether or not it was worth adding an extra 25-30 minutes to the study.

WB-MRI without diffusion detected 172 lesions distributed as 82 vertebral, 43 pelvic, 33 appendicular, 10 thoracic and 4 calvarial. Out of these lesions, 112 lesions were confidently reported metastatic with a distribution of 62 vertebral, 26 pelvic, 18 appendicular, 4 thoracic and 2 calvarial. The remaining 60 lesions were considered benign (44 lesions) or equivocal (16 lesions).

On adding diffusion, 197 lesions were detected with an extra of 25 lesions. 17 of these extra lesions were of significance proven metastatic ( 2 by biopsy and 15 by follow up) and 3 other lesions turned out false positive ( 2 by biopsy and 1 by follow up). The remainder 5 lesions were true negative for metastases. Those lesions were met with in 14 patients.

There was another mismatch but this time the lesions were seen on bone scan and not on MRI, they were 20 lesions on WB-MRI without diffusion and came down to 14 lesions on adding DWI.

It is worth mentioning that the majority of missed lesions (9) on MRI were in one case having diffusely accentuated red marrow signal attributed to chronic anaemia obscuring the lesions (metastasis was suspected by the reporter, but distinct specific lesions couldn't be verified) as demonstrated in figure (5).
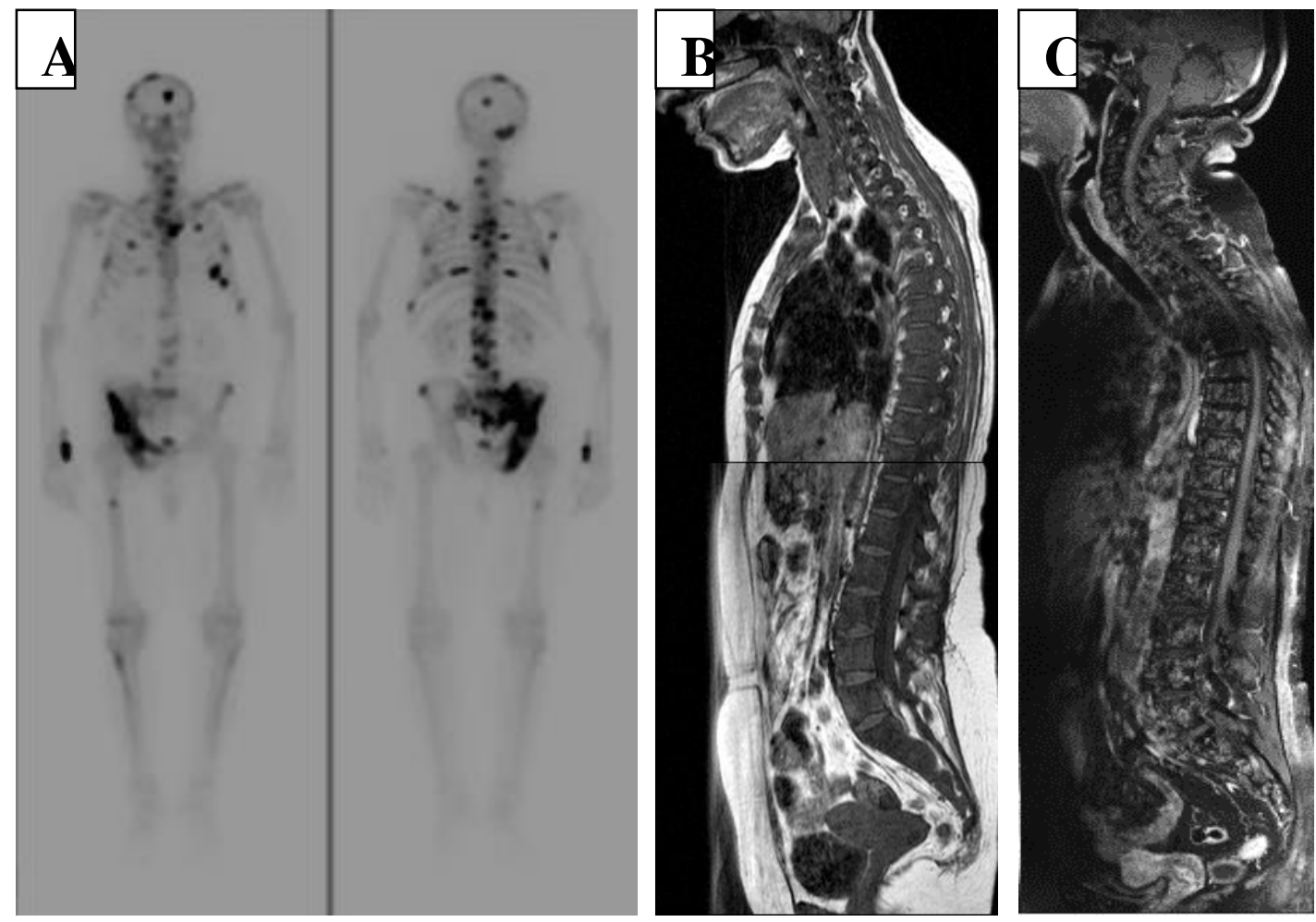

Figure (5): A known case of metastatic breast cancer. (A)Anterior and posterior projections bone scan showed a heavy metastatic load implicating the spine, pelvis and ribs. (B) Sagittal T1W image of the whole spine showed diffusely hypointense vertebral marrow with a sense of inhomogeneity, no definite lesions could be delineated. (C) Sagittal post contrast T1W image with fat suppression showed diffuse heterogeneity of contrast enhancement with equivocal lesions seen at the lumbar spine. This was a case with chronic anaemia and metastases on to. It was rather tricky on MRI. 
After establishment of the diagnosis through biopsy or follow up, on WB-MRI without diffusion 91 lesions were true positive, 18 lesions false positive, 34 lesions true negative, 14 lesions false negative and 16 lesions were equivocal. Then on WB-MRI with diffusion, 119 lesions were true positive, 13 lesions false positive, 41 lesions true negative, 11 lesions false negative and 9 lesions remained equivocal.

Bone scan has a higher accuracy for the detection and characterization of vertebral and calvarial lesions while it wouldn't be the modality of choice for appendicular lesions.

On the other hand, WB-MRI has a higher accuracy for the detection and characterization of pelvic, appendicular followed by vertebral lesions while it wouldn't be the modality of choice for calvarial lesions. As for thoracic lesions, we found out that DWI improved their detection through 3D MPR and thick stack coronal MIP's.

Globally, on comparing sensitivities; bone scan was $85.6 \%$, WB-MRI without diffusion was $73.4 \%$ and WB-MRI with diffusion $88.9 \%$. On comparing specificities; bone scan was $67.5 \%$, WBMRI without diffusion was $68.8 \%$ and WB-MRI with diffusion was $82.9 \%$. On comparing PPV's; bone scan was $61.7 \%$, WB-MRI without diffusion was $78.1 \%$ and WB-MRI with diffusion was $93 \%$. On comparing NPV's; bone scan was $66.3 \%$, WB-MRI without diffusion was $62.6 \%$ and WB-MRI with diffusion 69\%.[7]

We compared these results with other studies, the majority of which didn't employ diffusion studies and only included STIR and T1W sequences with and without contrast.

The first comparative study between whole-body MRI using turbo spin imaging and planar skeletal scintigraphy was carried out in 1997 by Eustace et al. A total of 25 patients with various tumor types were examined. The authors reported 96.5\% sensitivity and $100 \%$ specificity for whole body MRI. Planar skeletal scintigraphy as the established method had a sensitivity of $72 \%$ and a specificity of 98\%.[8]
Steinborn et al. examined 18 patients with various malignant primary diseases and suspicion of bone metastases with an examination protocol that incorporated STIR imaging and a T1-weighted spinecho sequence. The whole-body MRI detected $91.4 \%$ of the metastases that were verified during the course of the disease, whereas skeletal scintigraphy only revealed $85 \%$ of lesions.[9]

We found out that adding diffusion to conventional MR sequences improved the conspicuity of rib lesions, this was supported in Xian $\mathrm{Xu}$, et al's study that also concluded that DWI had no significant influence on the sensitivity of MRI for calvarial lesions.[10]

Despite the statistical values favouring DWI, we have to admit that establishing the diagnosis based on follow up is not a $100 \%$ accurate method especially in stationary lesions and regressive lesions without therapy but again the gold standard of histopathological verification is not feasible for all suspected metastatic lesions.

By incorporating DWI, our study could be considered a dual study going on parallel to each other comparing MRI as a multiparametric upgraded protocol with Bone scan and at the same time comparing the reproducibility of MRI with and without DWI in the detection of osseous and soft tissue metastases.

Assessment of lesions included qualitative assessment of lesions based on signal intensity on ADC maps, the more hypointense the lesion the more suggestive of cellular packing and thus of metastases and quantitative assessment through ADC values.

There were variable degrees of overlap in ADC values between the different pathological entities, the ones of significance are those intersecting with metastases, where there is an overlap between the Low ADC values of red marrow and metastasis at the range of 0.61 to $0.69 \times 10^{-3} \mathrm{~mm}^{2} / \mathrm{sec}$ and then between metastasis and other benign pathological marrow entities at the range of 0.9 to 1.03 $\mathrm{x} 10^{-3} \mathrm{~mm}^{2} / \mathrm{sec}$ sparing the midrange of 0.7 to 0.89 where only metastatic lesions gave these values in our study. 


\section{Wafaa Abdel Hamid}

Another study showed Mean ADCs of the normal bone marrow, $0.57 \pm 0.20$ and 0.29 $\pm 0.15 \times 10^{-3} \mathrm{~mm}^{2} / \mathrm{s}$, for red and yellow marrow respectively and a mean ADC value of $0.61+/-0.21 \times 10^{-3} \mathrm{~mm}^{2} / \mathrm{s}$ for metastatic lesions.[10]

Through all the studies - we came across that tackled quantitative assessments of diffusion through ADC values, there was no sole cut off value; each study showed a degree of overlap between normal marrow, benign and malignant lesions. $\mathrm{We}$ concluded in our study that a mean range of 0.7 to $0.89 \times 10^{-3} \mathrm{~mm}^{2} / \mathrm{sec}$ was found in metastatic lesions only but then relying on these values to call for metastases in any patient would be unwise because of the influence of underlying marrow being predominantly yellow or red and the lesion itself being lytic, sclerotic or mixed that could significantly alter these values.

Biopsy was accessible in 25 lesions, mainly undergone to verify descripancies between both modalities in lesions that would have an impact on management strategies.

Biopsy also played a role in this study to assess reliability of DWI as a sole diagnostic tool with lesions indistinguishable/inconspicuous on conventional MRI and bone scan.

The forementioned results are representing one sector of the study which is considered the main sector, but then there is another parallel sector of major influence on patient's management and therapeutic planning which is related to the detection and verification of soft tissue metastatic involvement and comparing results of MRI with and without DWI.

DWI was capable of detecting 6 more hepatic lesions. It added two more patients that were not diagnosed with hepatic metastases and thus influencing their therapeutic strategies.

An extra case with an adrenal metastasis was diagnosed only on DWI, this case had no other soft tissue metastatic lesions and so this input was of major importance in management planning.

A vast number of studies have assessed the role of DWI in the detection and characterization of hepatic and adrenal lesions.

Li et al compared DWI with standard T2W sequence in the detection and characterization of hepatic focal lesions revealing an overall detection rate (averaged for two observers) significantly higher for DW (87.7\%) versus T2weighted $(70.1 \%)$ imaging $(\mathrm{P}<.001)$. FLL characterization was not significantly different between DW (89.1\%) and T2weighted $(86.8 \%)$ imaging $(\mathrm{P}=.51)$. ADCs of malignant FLLs were significantly lower than those of benign FLLs $(\mathrm{P}<.001) .[11]$

An extra case with metastatic leptomeningeal infiltration in the base of the skull was only detected on DWI adding to patient's metastatic load. This patient showed dramatic disappearance of the leptomeningeal infiltration after 3 months post CTH.

The majority of soft tissue lesions were verified on follow up and only the left calf intramuscular lesions, the left renal metastasis and an adrenal metastasis were verified by biopsies.

Bone scan played a limited role in detection of soft tissue lesions with only one of the cases showing abnormal skin and soft tissue uptake at both calf regions. 


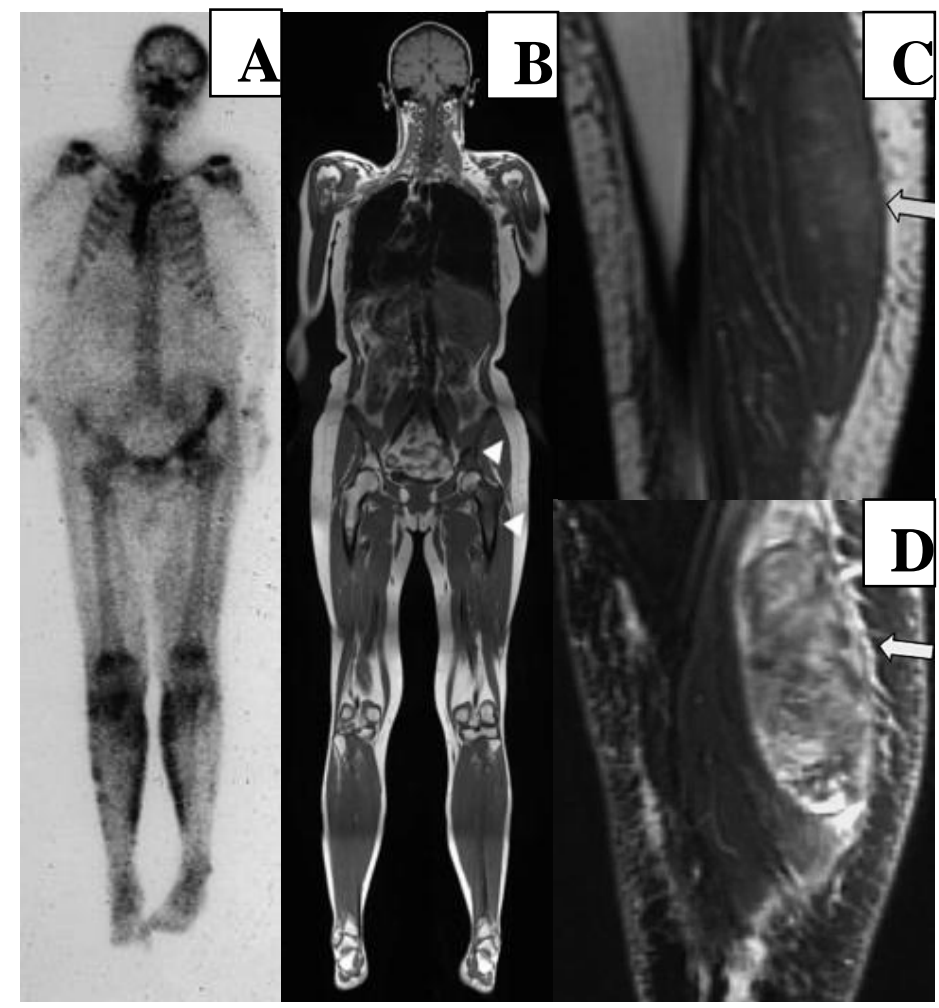

Figure (6): A case of lung cancer with paraneoplastic syndrome. (A) Anterior projection bone scan shows a metabolic superscan with non visualization of the kidneys and preferential diffuse hot uptake of the calvarium and sternum. There is abnormal hot uptake at left iliac bone and left femoral head and proximal shaft. There is also abnormal uptake at the skin of both legs (calf regions). (B) WB-MRI T1W coronal image showing marrow infiltration at left iliac bone and left femur (arrowheads). (C) sagittal T1W and (D) sagittal post contrast T1W with fat suppression images of the left calf region showing a heterogenous gastrocnemeus soft tissue lesion histopathologically confirmed to be metastatic.

Our attempt was to help place guidelines for oncologists in the triaging of cancer patients, as to which imaging modality they would benefit the most sparing them the loss of unnecessary time and money and pointing out choices that would directly influence their management planning and prognosis.

\section{CONCLUSION}

We don't think that WB-MRI should replace bone scan in the quest for skeletal metastases. However, we believe that WB-MRI should be the modality of choice when both skeletal and soft tissue metastases are suspected and in the follow up of a known metastatic patients for adequate coverage of both skeletal and soft tissue structures combined with qualitative and quantitative features of DWI helping assess tumors at cellular levels even prior to gross morphological changes verifying response to therapy as early as possible sparing patients precious time from drifting with delayed decision making. 


\section{Wafaa Abdel Hamid}

\section{REFERENCES}

1)

Resnick D, Stavros C \& Michael D. Histogenesis, Anatomy and Physiology. In: Donald Resnick (ed): Diagnosis of Bone and Joint Disorders (4 ${ }^{\text {th }}$ edition) 2002: 289-372.

2) N. Ghanem, C. Altehoefer, T. Kelly, et al. (February 2006)"Whole-body MRI in Comparison to Skeletal Scintigraphy in Detection of Skeletal Metastases in Patients with Solid Tumors" In Vivo 20(1): 173-182.

3) Altehoefer C, Ghanem N, Moser E and Langer M (2001) "Comparative detectability of bone metastases and impact on

therapy of magnetic resonance imaging and bone scintigraphy in

patients with breast cancer." Eur J Radiol 40: 16-23.

4) Steinborn MM, Heuck AF, Tiling R, et al. (2003): "Whole-body bone marrow MRI in patients with metastastic disease to skeletal system." J Comput Assit Tomogr. 23: 123-129.

5) Ghanem N, Altehoefer C, Scofer O, et al. (2003) "Whole-body MRI as a new screening and staging technique in detection of skeletal metastases in comparison to skeletal scintigraphy and whole-body FDG-PET. Garmisch 10th International MRI Symposium MRI: reinventing its novelty 45: 96-112.

6) Ryota Fujimoto, Tatsuya Higashi, Yuji Nakamoto, et al (2006) "Diagnostic accuracy of bone metastases detection in cancer patients: Comparison between bone scintigraphy and whole-body FDG-PET." Annals of Nuclear Medicine 20( 6): 399-408.
7)Gold RI, Seeger LL, Bassett LW, Steckel RJ.(March 2001) "An integrated approach to the evaluation of metastatic bone disease." Radiol Clin North Am. 28(2):471-83.

8) Eustace $S$, Tello $R$, DeCarvalho $V$, et al.(1997) " A comparison of whole-body turbo STIR MR imaging and planar 99m Tcmethylene diphosphonate scintigraphy in the examination of patients with suspected skeletal metastases." AJR Am J Roentgenol 169: 16551661.

9) Steinborn MM, Heuck AF, Tiling R, et al. (2001) "Whole-body bone marrow MRI in patients with metastastic disease to skeletal system." J Comput Assit Tomogr. 23: 123129.

10) Xian $\mathrm{Xu}$, Lin Ma, Jin-shan Zhang, et al. (September 2008) "Feasibility of Whole Body Diffusion Weighted Imaging in Detecting Bone Metastasis." Chinese Medical Sciences J. 23(3):

151-157.

11) Li S, Xue HD, Sun F and Jin ZY. (April 2009) "Feasibility and clinical value of whole body diffusion weighted magnetic resonance imaging in detection of bone metastases." Seminars of Radiology 31(2): 192-199. 